Feng Yi-Min, Tai Xi-Shi and Xia Yu-Pei*

\title{
The crystal structure of [(2,2'-bipyridine- $\left.\mathrm{k}^{2} N, N\right)$ - bis(6-phenylpyridine-2-carboxylate- $\mathrm{k}^{2} \mathrm{~N}, \mathrm{O}$ ) copper(II)], $\mathrm{C}_{34} \mathrm{H}_{24} \mathrm{~N}_{4} \mathrm{O}_{4} \mathrm{Cu}$
}

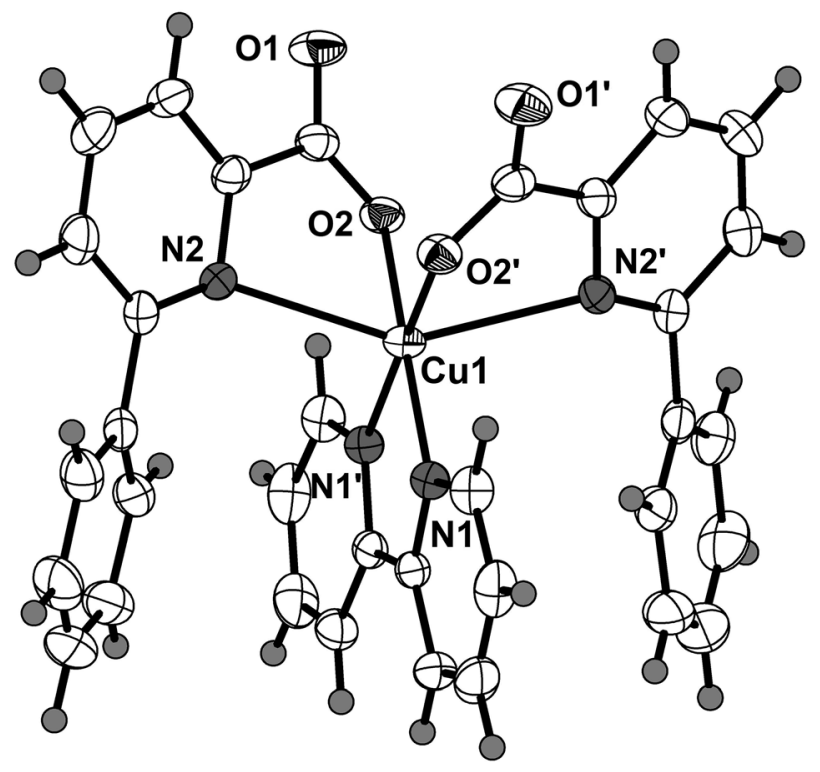

https://doi.org/10.1515/ncrs-2021-0486

Received December 23, 2021; accepted January 24, 2022; published online February 18, 2022

\begin{abstract}
$\mathrm{C}_{34} \mathrm{H}_{24} \mathrm{~N}_{4} \mathrm{O}_{4} \mathrm{Cu}$, monoclinic, $\mathrm{C2} / \mathrm{c}$ (no. 15), $a=17.2931(15) \AA$, $b=10.3584(5) \AA, c=17.5641(16) \AA, \beta=120.180(12)^{\circ}$, $V=2719.8(5) \AA^{3}, Z=4, R_{g t}(F)=0.0282, w R_{\text {ref }}\left(F^{2}\right)=0.0681$, $\mathrm{T}=199.99(10) \mathrm{K}$.
\end{abstract}

\section{CCDC no.: 2144052}

The molecular structure is shown in the figure. Table 1 contains crystallographic data and Table 2 contains the list of the atoms including atomic coordinates and displacement parameters.

*Corresponding author: Xia Yu-Pei, College of Chemistry and Chemical Engineering, Weifang University, Weifang, Shandong 261061, P. R. China, E-mail: yopoxia@126.com

Feng Yi-Min and Tai Xi-Shi, College of Chemistry and Chemical Engineering, Weifang University, Weifang, Shandong 261061, P. R. China. https://orcid.org/0000-0002-0050-1900 (T. Xi-Shi)

Ә Open Access. (c 2022 Feng Yi-Min et al., published by De Gruyter. (बc) BY International License.
Table 1: Data collection and handling.

\begin{tabular}{|c|c|}
\hline Crystal: & Blue block \\
\hline Size: & $0.14 \times 0.12 \times 0.09 \mathrm{~mm}$ \\
\hline Wavelength: & Mo $K \alpha$ radiation $(0.71073 \AA ̊)$ \\
\hline$\mu:$ & $0.85 \mathrm{~mm}^{-1}$ \\
\hline Diffractometer, scan mode: & SuperNova, $\omega$ \\
\hline$\theta_{\max }$, completeness: & $25.0^{\circ}, 99 \%$ \\
\hline$N(h k l)_{\text {measured }}, N(h k l)_{\text {unique }}, R_{\text {int }}:$ & $6381,2368,0.023$ \\
\hline Criterion for $I_{\mathrm{obs}}, N\left(h k l_{\mathrm{gt}}\right.$ : & $I_{\text {obs }}>2 \sigma\left(I_{\text {obs }}\right), 2201$ \\
\hline$N(\text { param })_{\text {refined: }}$ & 195 \\
\hline Programs: & $\begin{array}{l}\text { Bruker [1], CrysAlis }{ }^{P R O}[2] \text {, } \\
\text { Olex2 [3], SHELX [4], Diamond [5] }\end{array}$ \\
\hline
\end{tabular}

\section{Source of material}

The mixture of 6-phenylpyridine-2-carboxylic acid $(0.50 \mathrm{mmol}, 0.0996 \mathrm{~g}), 2,2^{\prime}$-bipyridine $(0.25 \mathrm{mmol}$, $0.039 \mathrm{~g}), \mathrm{NaOH}(0.50 \mathrm{mmol}, 0.020 \mathrm{~g})$, and cupric acetate monohydrate $(0.25 \mathrm{mmol}, 0.0499 \mathrm{~g})$ were dissolved in a $15 \mathrm{~mL}$ water $(5 \mathrm{~mL})$-ethanol $(10 \mathrm{~mL})$ solution at room temperature. The blue solution gradually became cloudy. The reaction mixture was stirred for $4 \mathrm{~h}$ at $75^{\circ} \mathrm{C}$, cooled to room temperature and stirring was continued for $4 \mathrm{~h}$. Then the solution was filtered and the filtrate was left resting for 20 days. The blue crystals of the title complex were obtained from the filtrate.

\section{Experimental details}

The hydrogen atoms were positioned geometrically $(\mathrm{C}-\mathrm{H}=0.93 \AA)$. Their $U_{\text {iso }}$ values were set to $1.2 U_{\text {eq }}$ of the parent atoms.

\section{Comment}

As one of the important research contents of coordination chemistry, the complexes have been widely valued by chemists and material scientists. Because they show a rich variety of structures [6] and many applications such as 
Table 2: Fractional atomic coordinates and isotropic or equivalent isotropic displacement parameters $\left(\AA^{2}\right)$.

\begin{tabular}{lrrrr}
\hline Atom & $\boldsymbol{x}$ & $\boldsymbol{y}$ & $\boldsymbol{z}$ & \multicolumn{1}{c}{$\boldsymbol{U}_{\text {iso }} / \boldsymbol{U}_{\text {eq }}$} \\
\hline Cu1 & 0.500000 & $0.57566(3)$ & 0.750000 & $0.02216(11)$ \\
O1 & $0.36127(10)$ & $0.25220(13)$ & $0.70883(11)$ & $0.0391(4)$ \\
O2 & $0.42283(9)$ & $0.44497(12)$ & $0.75858(9)$ & $0.0275(3)$ \\
N1 & $0.57369(10)$ & $0.72313(14)$ & $0.74800(10)$ & $0.0222(3)$ \\
N2 & $0.39380(10)$ & $0.50940(15)$ & $0.59600(10)$ & $0.0238(3)$ \\
C1 & $0.38453(12)$ & $0.53909(19)$ & $0.51727(13)$ & $0.0266(4)$ \\
C2 & $0.36522(15)$ & $0.4455(2)$ & $0.45335(14)$ & $0.0373(5)$ \\
H2 & 0.361309 & 0.467595 & 0.400212 & $0.045^{*}$ \\
C3 & $0.35196(15)$ & $0.3198(2)$ & $0.46951(15)$ & $0.0416(6)$ \\
H3 & 0.338934 & 0.256146 & 0.427421 & $0.050^{*}$ \\
C4 & $0.35822(14)$ & $0.2894(2)$ & $0.54894(15)$ & $0.0342(5)$ \\
H4 & 0.347741 & 0.205658 & 0.560623 & $0.041^{*}$ \\
C5 & $0.38041(12)$ & $0.38610(18)$ & $0.61069(13)$ & $0.0251(4)$ \\
C6 & $0.38899(13)$ & $0.35681(18)$ & $0.69949(13)$ & $0.0267(4)$ \\
C7 & $0.54308(13)$ & $0.84129(17)$ & $0.75096(12)$ & $0.0226(4)$ \\
C8 & $0.59072(14)$ & $0.9520(2)$ & $0.75708(13)$ & $0.0317(5)$ \\
H8 & 0.568612 & 1.032979 & 0.759100 & $0.038^{*}$ \\
C9 & $0.67149(15)$ & $0.9398(2)$ & $0.76012(15)$ & $0.0395(6)$ \\
H9 & 0.704456 & 1.012913 & 0.763969 & $0.047^{*}$ \\
C10 & $0.70340(14)$ & $0.8190(2)$ & $0.75746(14)$ & $0.0367(5)$ \\
H10 & 0.757763 & 0.809301 & 0.759262 & $0.044^{*}$ \\
C11 & $0.65292(13)$ & $0.7129(2)$ & $0.75208(13)$ & $0.0294(5)$ \\
H11 & 0.674625 & 0.631087 & 0.751235 & $0.035^{*}$ \\
C12 & $0.36034(14)$ & $0.7711(2)$ & $0.53443(13)$ & $0.0337(5)$ \\
H12 & 0.340466 & 0.747001 & 0.572719 & $0.040^{*}$ \\
C13 & $0.39046(13)$ & $0.67742(19)$ & $0.49878(13)$ & $0.0284(4)$ \\
C14 & $0.42071(14)$ & $0.7158(2)$ & $0.44225(14)$ & $0.0372(5)$ \\
H14 & 0.440530 & 0.654206 & 0.417394 & $0.045^{*}$ \\
C15 & $0.42138(16)$ & $0.8446(2)$ & $0.42301(16)$ & $0.0471(6)$ \\
H15 & 0.442714 & 0.869365 & 0.386038 & $0.057^{*}$ \\
C16 & $0.39102(18)$ & $0.9365(2)$ & $0.45766(17)$ & $0.0509(7)$ \\
H16 & 0.391380 & 1.023087 & 0.443921 & $0.061^{*}$ \\
C17 & $0.35973(17)$ & $0.9000(2)$ & $0.51327(16)$ & $0.0454(6)$ \\
H17 & 0.338355 & 0.962065 & 0.536352 & $0.054^{*}$ \\
\hline & & & &
\end{tabular}

antiepileptic drug, fluorescent sensor, photophysical property, anticancer property, and optical property [7-10]. Previous studies from our research group showed that both 6-phenylpyridine-2-carboxylate acid and $2,2^{\prime}$-bipyridine are both excellent ligands. Some ternary metal complexes constructed by $\mathrm{Pb}(\mathrm{II}), \mathrm{Zn}(\mathrm{II})$, and $\mathrm{Co}(\mathrm{II})$ with 6-phenylpyridine-2-carboxyate acid ligand have been reported [11-13]. In order to continue our study a new $\mathrm{Cu}$ (II) complex $\left[\left(2,2^{\prime}\right.\right.$-bipyridine- $\left.\mathrm{k}^{2} N, N\right)$-bis(6-phenylpyridine-2-carboxylate- $\left.\mathrm{k}^{2} N, O\right)$ copper(II)] has been synthesized and structural characterized.

The asymmetric unit consists of one half of a $\mathrm{Cu}(\mathrm{II})$ ion, one 6-phenylpyridine-2-carboxylate ligands, and one half of a 2,2'-bipyridine ligand (see the figure). The title complex is located on a two fold axis. all ligands adopt the bischelating coordination mode. The coordinated environment of $\mathrm{Cu}$ (II) center is shown in the Figure (symmetry code: ' $1-x,+y, 3 / 2-z)$. The $\mathrm{Cu}(\mathrm{II})$ ion locates in a distorted octahedral geometry, defined by one $\mathrm{O}$ atom $(\mathrm{O} 2)$ form one 6-phenylpyridine-2-carboxylate ligand and one $\mathrm{N}$ atom (N1) from 2,2'-bipyridine ligand at the axial positions ( $\mathrm{O} 2-\mathrm{Cu} 1-$ $\left.\mathrm{N} 1,173.37(6)^{\circ}\right)$, one $\mathrm{O}$ atom $\left(\mathrm{O}^{\prime}{ }^{\prime}\right)$ and two $\mathrm{N}$ atoms (N2, $\mathrm{N}^{\prime}$ ) form 6-phenylpyridine-2-carboxylate ligand, and one $\mathrm{N}$ atom $\left(\mathrm{N1}^{\prime}\right)$ from the 2,2'-bipyridine ligand in the equatorial plane. The dihedral angle between benzene ring (C12-C13$\mathrm{C} 14-\mathrm{C} 15-\mathrm{C} 16-\mathrm{C} 17)$ and pyridine ring $(\mathrm{C} 1-\mathrm{C} 2-\mathrm{C} 3-\mathrm{C} 4-\mathrm{C} 5-\mathrm{N} 2)$ of 6-phenylpyridine-2-carboxylate ligand is $33.4^{\circ}$. The crystal packing is stabilized by the $\pi-\pi$ stacking to form a 3D network structure.

Author contributions: All the authors have accepted responsibility for the entire content of this submitted manuscript and approved submission.

Research funding: This project was supported by the National Natural Science Foundation of China (No. 21171132), the Natural Science Foundation of Shandong (ZR2014BL003), the Project of Shandong Province Higher Educational Science and Technology Program (J14LC01) and Science Foundation of Weifang.

Conflict of interest statement: The authors declare no conflicts of interest regarding this article.

\section{References}

1. Bruker. SAINT and SADABS; Bruker AXS Inc.: Madison, Wisconsin, USA, 2000.

2. Rigaku Oxford Diffraction. CrysAlisPRO Software system (version 1.171.38.43f); Rigaku Corporation: Oxford, UK, 2015.

3. Dolomanov O. V., Bourhis L. J., Gildea R. J., Howard J. A. K., Puschmann H. OLEX2: a complete structure solution, refinement and analysis program. J. Appl. Crystallogr. 2009, 42, 339-341.

4. Sheldrick G. M. Crystal structure refinement with SHELXL. Acta Crystallogr. 2015, C71, 3-8.

5. Brandenburg K. DIAMOND. Visual Crystal Structure Information System (version. 3.2); Crystal Impact: Bonn, Germany, 2012.

6. Lv B.-L. Crystal structure of catena-poly[aqua-(4-iodopyridine2,6-dicarboxylato- $\left.\kappa^{3} N, O, O^{\prime}\right)-\left(\mu_{2}-4\right.$-amino-4H-1,2,4-triazole- $\left.\kappa^{2} N: \mathrm{N}^{\prime}\right)$ copper(II)], $\mathrm{C}_{9} \mathrm{H}_{8} \mathrm{~N}_{5} \mathrm{O}_{5} \mathrm{Cul}$. Z. Kristallogr. NCS 2021, 236, 873-875.

7. Mahmoud M.-A., Abbas A.-M., Zaitone S.-A., Ammar A.-M., Sallam S.-A. Copper(II) ternary complexes with gabapentin and neurotransmitters as antiepileptic drug. J. Mol. Struct. 2019, 1180, 861-877.

8. Liu S.-Y., Tian J.-J., Zhu L.-J., Tian H.-T., Yang M., Huang K.-L., Xu W. - T. A rapid fluorescent ratiometric $\mathrm{Ag}+$ sensor based on synthesis of a dual-emission ternary nucleotide/terbium complex probe. Microchem. J. 2021, 170, 106658.

9. Al-Busaidi I.-J., Ilmi1 R., Zhang D.-Y., Dutra J.-D.-L., Oliveira W.-F., Rasbi N.-K.-A., Zhou L., Wong W.-Y., Raithby P.-R., Khan M.-S. Synthesis and photophysical properties of ternary $\beta$-diketonate europium(III) complexes incorporating bipyridine and its derivatives. Dyes Pigments 2022, 197, 109879.

10. Chew H.-N., Thean H.-T., Ngee H.-T., Hoi L.-S., Munirah A., Seik W.-N., Wei K.-G., May L.-L., Jing W.-L., Mohd Z. Synthesis, 
characterization and multiple targeting with selectivity: anticancer property of ternary metal phenanthroline-maltol complexes. J. Inorg. Biochem. 2021, 220, 111453.

11. Tai X.-S., Liang L., Li X.-T., Cao S.-H., Wang L.-H. Crystal structure of diaqua-bis $\left(\mu_{2}-6\right.$-phenylpyridine-2-carboxylate- $\left.\kappa^{3} \mathrm{~N}, \mathrm{O}: 0\right)$ bis(6-phenylpyridine-2-carboxylato- $\kappa^{2} \mathrm{~N}, \mathrm{O}$ ) lead(II)- $\mathrm{N}, \mathrm{N}$ dimethylformamide-water (1/2/4), $\mathrm{C}_{54} \mathrm{H}_{58} \mathrm{~N}_{6} \mathrm{O}_{16} \mathrm{~Pb}_{2}$. Z. Kristallogr. NCS 2021, 236, 1199-1201.
12. Tai X.-S., Wang Z.-J., Ouyang J., Li Y.-F., Zhang W., Jia W.-L., Wang L.-H. The crystal structure of [(phenantroline- $\kappa^{2} N, N^{\prime}$ )-bis(6-phenylpyridine2-carboxylate- $\kappa^{2} N, O$ ) cobalt(II)]monohydrate, $\mathrm{C}_{36} \mathrm{H}_{26} \mathrm{~N}_{4} \mathrm{O}_{5} \mathrm{Co}$. Z. Kristallogr. NCS 2021, 236, 1309-1311.

13. Wang L.-H., Wang Z.-J., Ouyang J., Tai X.-S. The crystal structure of bis(6-phenylpyridine-2-carboxylate- $\left.\kappa^{2} N, O\right)$-(2,2'-bipyridine$\left.\kappa^{2} N, N^{\prime}\right)$ zinc(II) monohydrate, $\mathrm{C}_{34} \mathrm{H}_{26} \mathrm{~N}_{4} \mathrm{O}_{5} \mathrm{Zn}$. Z. Kristallogr. NCS 2021, 236, 1297-1299. 